Maria do Rosário Mascaro Machado ${ }^{1}$ Fernando de Almeida Machado²

${ }^{1}$ Coordenadora do Núcleo de Vigilância Epidemiológica do Hospital Geral de Palmas. Secretaria Estadual de Saúde, Palmas, TO, Brasil.

2 Professor Adjunto da Faculdade de Medicina da Universidade Federal do Tocantins, Palmas, TO, Brasil.

* Trabalho apresentado na forma de Comunicação Oral por Maria do Rosário Mascaro Machado, na VIII Mostra Nacional de Experiências Bem-Sucedidas em Epidemiologia, Prevenção e Controle de Doenças (Expoepi), novembro de 2008, Brasília, DF.

Trabalho não subvencionado.

Contato:

Maria do Rosário Mascaro Machado

Hospital Geral de Palmas/Núcleo de Vigilância Epidemiológica: 201 Sul, NS 01, Conjunto 2, Palmas-TO

CEP 77015.202

E-mail:

nucleo.hgp@saude.to.gov.br

\section{Acidentes com material biológico em trabalhadores de enfermagem do Hospital Geral de Palmas (TO)*}

\author{
Work-related accidents with biological material among nursing \\ staff from General Hospital in Palmas, state of Tocantins, Brazil
}

\section{Resumo}

Objetivo: identificar os acidentes de trabalho com material biológico ocorridos com os trabalhadores de enfermagem do Hospital Geral de Palmas (HGP) e verificar a ocorrência de subnotificação no Serviço de Segurança do Trabalho (SST) do HGP. Método: estudo descritivo transversal, com dados coletados por meio de um questionário autoaplicável que foi respondido por 389 profissionais de enfermagem (91\% do efetivo) nos meses de agosto e setembro de 2007. Resultados: dentre os profissionais de enfermagem, 178 (45,7\%) declararam já ter sofrido acidente com material biológico (55,6\% por acidente perfurocortante e 44,4\% com fluidos), sendo 106 (59,9\%) técnicos de enfermagem, 57 (32\%) auxiliares de enfermagem e $15(8,4 \%)$ enfermeiros. Considerando todos os profissionais de enfermagem $(n=389)$, com ou sem antecedente de acidente, os auxiliares constituíram o grupo mais exposto aos riscos de acidentes (54,3\%), seguidos pelos técnicos $(49,8 \%)$ e pelos enfermeiros (21,1\%). Dos 178 profissionais que sofreram acidentes, 95 (53,3\%) trabalhavam de 1 a 5 anos e 39 (21,9\%) há mais de 10 anos. Dentre os 178 profissionais acidentados, apenas 64 registraram o acidente no SST, resultando em $64 \%$ de subnotificação. Conclusão: estes resultados evidenciam a necessidade de reformulação do protocolo de encaminhamento para o atendimento ao acidentado e a participação efetiva dos profissionais de enfermagem nessa reformulação.

Palavras-chave: acidente de trabalho; equipe de enfermagem; acidente com perfurocortante.

\begin{abstract}
Objective: To identify work-related accidents with biological material among the nursing staff of General Hospital of Palmas (HGP) in Tocantins state, Brazil, and to assess underreporting in its Worker's Safety and Health Service (SST). Method: Descriptive cross-sectional study that used a self-reported questionnaire answered by 91\% of the nursing staff (389 nurses) between August and September 2007. Results: One hundred and seventy eight (45.7\%) nurses reported accidental exposure to biological material, caused by puncture or lacerating objects (55.6\%) and by fluids (44.4\%). One hundred and six (59.9\%) of them were nursing assistants, 57 (32\%) were healthcare assistants, and 15 (8.4\%) were nurses. Considering the whole nursing staff ( $n=389)$, including both who had and who did not have accidents, healthcare assistants were the ones who most frequently suffered such accidents (54.3\%), followed by the nursing assistants (49.8\%) and nurses (21.1\%). Among 178 professionals accidentally exposed to biological material, 95 (53.3\%) had worked between one to five years in the hospital, while 39 (21.9\%), for more than 10 years. Out of 178, only 64 accidents were recorded at the SST, resulting in $64 \%$ of underreporting. Conclusion: these findings indicated the need to reformulate the procedures for reporting and assisting the injured workers, with the effective participation of nursing staff in this reformulation.
\end{abstract}

Keywords: occupational injuries; nursing staff; accidental puncture and laceration. 


\section{Introdução}

Entende-se por acidentes de trabalho, eventos bem configurados no tempo e no espaço cujas consequências, imediatas em grande parte dos casos, permitem estabelecer o nexo causal com o trabalho. O Ministério da Previdência e Assistência Social define como acidente de trabalho o ocorrido durante exercício do trabalho ou no trajeto a serviço da empresa, o qual provoca lesão corporal ou perturbação funcional que cause a morte, a perda ou a redução, permanente ou temporária, da capacidade para o trabalho (BRASIL, 2000).

Os profissionais de saúde estão constantemente sob risco de sofrer acidentes ocupacionais através de exposição aos diferentes agentes veiculados pelo sangue e outros fluidos orgânicos (BRASIL, 2004; RAPPARINI et al., 2007; SOERENSEN et al., 2009). O ambiente de trabalho hospitalar é considerado uma grande área de risco de acidentes, sejam eles causados por agentes biológicos, químicos, físicos, psicossociais ou decorrentes da organização do trabalho (SUAZO, 1999; NISHIDE; BENATTI; ALEXANDRE, 2004; GUILARDE et al., 2010).

As exposições do trabalhador a material biológico podem ser percutâneas ou mediante contato com membranas mucosas. Qualquer contato direto com material laboratorial potencialmente contaminado por microrganismos é também considerado uma exposição e requer avaliação (CENTERS FOR DISEASE CONTROL AND PREVENTION, 2005; CANALLI, 2008). Dentre os fluidos corporais, tem-se reconhecido o sangue como o mais importante veículo de transmissão ocupacional dos vírus das hepatites $\mathrm{B}$ e $\mathrm{C}$ e HIV (CIORLIA; ZANETTA, 2004; SPAGNUOLO; BALDO; GUERRINI, 2008). Sabe-se que a prevalência sorológica do vírus da hepatite B (VHB) nos trabalhadores de saúde é elevada, em torno de três a cinco vezes maior que a encontrada na população em geral. Comparado com outras doenças, o risco de adquirir hepatite B é cerca de 100 vezes maior que o risco de conversão sorológica pelo HIV e 10 vezes maior que o risco para o vírus da hepatite C (VHC), dependendo das características do acidente (BRASIL, 2001; RESENDE; FORTALEZA, 2001; BRAGA, 2005). Estudos prospectivos estimam que o risco de transmissão do HIV, após exposição de membrana mucosa a fluidos, é de 0,09 por cento, enquanto após acidentes perfurocortantes é de 0,3 a 0,5 por cento (TEIXEIRA; SANTOS, 1999; RAPPARINI, 2002).

Em 1985, os Centers of Disease Control and Prevention (CDC), dos EUA, introduziram a necessidade da adoção de medidas de proteção, conhecidas como precauções universais, depois reformuladas para precauções padrão. Essas precauções preconizam recomendações a serem observadas com a finalidade de reduzir os riscos de transmissão de microrganismos de fonte conhecidas ou não para os trabalhadores, devendo ser utilizadas na assistência a todos os pacientes (GARNER, 1996). É necessário destacar que as precauções padrão podem ajudar a reduzir, mas não eliminam o risco de exposição ocupacional (SHIMUZU; RIBEIRO, 2002).

Embora muitas instituições tenham adotado as precauções padrão como medidas de proteção aos trabalhadores, vários estudos mostram que a exposição e a infecção continuam ocorrendo de maneira elevada entre estudantes e profissionais de saúde em diferentes instituições nacionais (BALSAMO; BARRIENTO; ROSSI, 2000; GALON; ROBAZZI; MARZIALE, 2008; CANALLI; MORIYA; HAYASHIDA, 2010). Somando-se a esta preocupação, observa-se também a subnotificação do acidente (FACCHIN, 2009). Enquanto os profissionais de saúde não se conscientizarem da importância da notificação do acidente, a dimensão real do problema não poderá ser determinada.

Quando se consegue coletar todas as informações a respeito de um acidente, podem-se analisar os dados para priorizar esforços e estabelecer metas em unidades de maior risco, identificando as causas, no sentido de determinar se as ocorrências requerem aperfeiçoamento técnico na realização dos procedimentos, na aquisição de dispositivos mais seguros, na mudança de comportamento de funcionários ou se há falhas no suprimento de equipamentos de proteção individual e coletiva.

Diante da relevância do assunto, decidiu-se investigar os acidentes com material biológico ocorridos com os profissionais de enfermagem que trabalham no Hospital Geral de Palmas (HGP), capital do estado do Tocantins. Para isso, foi feito um estudo epidemiológico com os objetivos de identificar os acidentes de trabalho com material biológico ocorridos com os trabalhadores de enfermagem do HGP e verificar a ocorrência de subnotificação de acidentes com material biológico no Serviço de Segurança do Trabalho (SST) deste mesmo hospital.

\section{Metodologia}

Para o desenvolvimento desta pesquisa, realizou-se um estudo epidemiológico descritivo transversal. Os dados foram coletados pela pesquisadora por meio de um questionário autoaplicável. O questionário contém dados sociodemográficos, questões sobre a ocorrência de acidentes de trabalho com material biológico, preocupação do profissional com adoecimento decorrente do acidente e realização de notificação do acidente. Foram estudados acidentes ocorridos entre o período de agosto de 2005 a setembro de 2007 por serem essas datas, respectivamente, a da inauguração do HGP e do último mês de aplicação do questioná- 
rio. Os dados foram coletados nos meses de agosto e setembro de 2007, no local de trabalho, nos quatro turnos de serviço. Para análise dos dados, foi utilizado o programa Epi-Info 1996. O projeto obteve a aprovação do Comitê de Ética Médica do HGP e do Comitê de Ética em Pesquisa da Escola Nacional de Saúde Pública Sérgio Arouca da Fundação Oswaldo Cruz. Os participantes assinaram um Termo de Consentimento Livre e Informado. Os questionários não tiveram identificação dos respondentes.

O HGP Dr. Francisco Aires da Silva foi inaugurado em agosto de 2005. É uma instituição pública de administração estadual situada na cidade de Palmas que atende diversas especialidades, com 243 leitos (194 leitos nos dois andares do hospital, 18 leitos na UTI adulto, 5 leitos em UTI pediátrica, 18 no pronto socorro e 8 para atendimentos na hemodiálise) e capacidade para atender aproximadamente 4.500 pacientes/ mês. Segundo o Departamento de Pessoal, o Hospital conta com 997 funcionários sob gestão estadual e 378 funcionários terceirizados. É o maior hospital do Estado do Tocantins, com maior contingente de equipe de enfermagem num só lugar, motivo da escolha para execução deste trabalho.

Deste quadro de funcionários, há 74 enfermeiros, 26 técnicos de enfermagem e 329 auxiliares de enfermagem, totalizando 429 trabalhadores na equipe de enfermagem. Entre auxiliares de enfermagem, alguns declararam que exercem, na prática, a função de técnicos de enfermagem e, como tais, responderam ao questionário aplicado. A escolha da equipe de enfermagem para esta pesquisa se deu em função da elevada magnitude do número de acidentes de trabalho com material biológico e da suspeita da existência de subnotificação, o que tem sido documentado na literatura (FACCHIN, 2009), e por serem esses trabalhadores os que dispensam cuidado direto ao paciente e, consequentemente, possuem constantes riscos de exposição ocupacional.

Em um segundo momento, o SST do HGP foi procurado com o objetivo de obter as notificações de acidentes com material biológico da equipe de enfermagem referente ao mesmo período, ou seja, de agosto de 2005 a setembro de 2007. O SST é o responsável por fazer a notificação e o acompanhamento do funcionário acidentado. Essas notificações ficam arquivadas nesse serviço.

\section{Resultados}

Dos 429 funcionários da equipe de enfermagem do HGP, 389 participaram da pesquisa, o que representa 91\%.

Em relação às características gerais dos participantes (Tabela 1), observou-se que $89,2 \%$ são do sexo feminino, $213(54,7 \%)$ declararam-se técnicos de enfermagem, $105(27,0 \%)$ auxiliares de enfermagem e 71 (18,3\%) enfermeiros. O maior número de técnicos de enfermagem, em comparação com as informações obtidas no setor administrativo do hospital (apresentadas no item anterior) se explica porque muitos auxiliares exercem a função de técnicos e responderam ao questionário segundo as funções realmente executadas. A idade dos profissionais variou de 19 a 69 anos, apresentando uma média de 33,7 anos e mediana de 32,0 anos.

Tabela 1 Variáveis sociodemográficas e ocupacionais referentes aos profissionais de enfermagem $(\mathrm{N}=$ 389) do Hospital Geral de Palmas, TO, 2007

\begin{tabular}{llcc}
\hline Características & & $N$ & $\%$ \\
\hline \multirow{3}{*}{ Categoria profissional autorreferida } & Auxiliar de enfermagem & 105 & 27,0 \\
& Técnico de enfermagem & 213 & 54,7 \\
& Enfermeiro & 71 & 18,3 \\
& Masculino & 37 & 9,5 \\
Sexo & Feminino & 347 & 89,2 \\
& Não responderam & 5 & 1,3 \\
& & & \\
& 19 a 29 anos & 116 & 29,8 \\
Faixa etária & 30 a 39 anos & 181 & 46,6 \\
& 40 a 49 anos & 69 & 17,7 \\
& 50 a 59 anos & 9 & 2,3 \\
& 60 a 69 anos & 1 & 0,3 \\
& Não responderam & 13 & 3,3 \\
\hline
\end{tabular}


Verificou-se que $11,3 \%$ dos funcionários tinham menos de um ano de trabalho na profissão, 50,0\% com tempo de trabalho de 1 a 5 anos, 17,7\% com 5 a 10 anos e 21,0\% com mais de 10 anos (Tabela 2).

Dentre os funcionários participantes, 178 (45,7\%) declararam que já sofreram exposição acidental com material biológico durante suas atividades profissionais no HGP (Tabela 2). Dos 178 acidentados, 99 referiram ter sofrido acidente perfurocortante e 79 , acidentes com fluidos, sendo que $97(54,4 \%)$ declararam ter feito notificação escrita ou verbal sobre o seu acidente e $81(45,6 \%)$ afirmaram não ter feito nenhum tipo de notificação. Dos 178 profissionais, 165 (92,7\%) são do sexo feminino, $11(6,2 \%)$ são do sexo masculino e 2 $(1,1 \%)$ não responderam sobre o gênero.

Responderam que se preocupam em adquirir alguma doença através da exposição acidental, 372 profissionais (95,6 \%), sendo as mais citadas, em ordem de frequência, a AIDS $(57,8 \%)$, hepatites $(16,4 \%)$, todas $(13,1 \%)$, tuberculose $(4,1 \%)$, meningite $(3,6 \%)$, outras $(0,04 \%)$ e $4,3 \%$ não responderam.

Ao se fazer comparações dentro de cada categoria, observa-se que os auxiliares de enfermagem constituem o grupo mais exposto aos riscos de acidentes, visto que $54,3 \%$ de seus membros responderam positivamente ao antecedente de acidente. Na mesma análise, 49,8\% dos técnicos referiram ter sofrido algum acidente e apenas $21,1 \%$ dos enfermeiros fizeram tal referência. Entretanto, a comparação entre as três categorias apresentadas aponta que, dos 178 profissionais que sofreram a exposição a material biológico, 106 (59,9\%) são técnicos de enfermagem, 57 (32,0\%) são auxiliares de enfermagem e 15 $(8,4 \%)$ são enfermeiros.

Considerando o tempo de atividade profissional (anos de serviço na área de enfermagem), dos 178 profissionais que sofreram exposição, 16 (9,0\%) trabalhavam há menos de 1 ano, $95(53,3 \%)$ trabalhavam de 1 a 5 anos; 28 (15,7\%) de 5 a 10 anos; e $39(21,9 \%)$ a mais de 10 anos.
Dos 99 funcionários que sofreram perfuração, 59 são técnicos de enfermagem, 30 auxiliares de enfermagem e 10 enfermeiros. Desses, 75 (75,5\%) fizeram a notificação escrita ou verbal e $24(24,5 \%)$ não fizeram nenhuma notificação. Os profissionais com menos de 1 ano de trabalho foram os que menos se acidentaram ( $8 \% ; n=8)$, enquanto os que haviam trabalhado um período de 1 a 5 anos foram os que mais sofreram esse tipo de acidente $(52,5 \% ; n=52)$; funcionários entre 5 e 10 anos de atividade profissional totalizaram $15,5 \%(\mathrm{n}=15)$ e os com mais de 10 anos, $24,2 \%(\mathrm{n}=24)$.

Dos 79 profissionais que sofreram exposição acidental a fluidos corpóreos, 47 (59,4\%) são técnicos de enfermagem, 27 (34,2\%) auxiliares de enfermagem e $5(6,3 \%)$ enfermeiros. Desse total, 22 $(27,8 \%)$ fizeram a notificação e $57(72,2 \%)$ não fizeram notificação nem verbal, nem escrita do acidente. Nesse tipo de acidente, também os funcionários que trabalham há menos de 1 ano foram os que percentualmente menos se acidentaram $(10,1 \%$; $n$ =8); da mesma forma, os que mais se acidentaram foram os que trabalham de 1 a 5 anos (54,4\%; n = 43); funcionários entre 5 e 10 anos de atividade profissional totalizaram $16,4 \%(n=13)$ e os com mais de 10 anos, 19,0\% ( $\mathrm{n}=15)$.

De acordo com relatório do Serviço de Segurança do Trabalho do HGP (SSTHGP), de agosto de 2005 a setembro de 2007 foram notificados apenas 64 casos de acidentes com exposição a material biológico na equipe de enfermagem daquele hospital, distribuídos da seguinte forma: 55 acidentes com perfurocortantes $(85,9 \%)$ e 9 acidentes com fluidos corpóreos $(14,0 \%)$. Ainda de acordo com o registro do SSTHGP, dentre as notificações formalizadas, 31 (48,4\%) são de auxiliares, 26 (40,6\%) são de técnicos e 7 (10,9\%) de enfermeiros. Esses números mostram que o número de funcionários que afirmaram ter notificado $(\mathrm{n}=97)$ supera o número de notificações de fato registradas no serviço competente $(\mathrm{n}=64)$.

Tabela 2 Exposição acidental com material biológico, segundo o tempo de serviço, relatada por funcionários de enfermagem $(\mathrm{N}=388)$ do Hospital Geral de Palmas, TO, 2007

\begin{tabular}{lccc}
\hline Tempo de serviço (anos) & Acidentados & Não acidentados & Total \\
\hline Menos de 1 & $16(36,4 \%)$ & $28(63,6 \%)$ & 44 \\
De 1 a 5 & $95(48,9 \%)$ & $99(51,0 \%)$ & 194 \\
De 5 a 10 & $28(40,6 \%)$ & $41(59,4 \%)$ & 69 \\
Mais de 10 & $39(48,1 \%)$ & $42(51,9 \%)$ & 81 \\
Total & $178(45,9 \%)$ & $210(54,1 \%)$ & 388 \\
\hline
\end{tabular}




\section{Discussão}

Observa-se, neste trabalho, a predominância do profissional do sexo feminino nos acidentes com exposição a material biológico (92,7\%), resultado semelhante aos da literatura (CANINI, 2000; CIORLIA; ZANETTA, 2004; GUILARDE et al., 2010). A primeira explicação para os acidentes acontecerem mais entre as mulheres está no fato de a maioria dos trabalhadores da saúde ser do sexo feminino. Além disso, de maneira geral, a mulher insere-se no mercado de trabalho com o objetivo de contribuir para o aumento da renda familiar, frequentemente assumindo dupla ou tripla jornada de trabalho, o que favorece o desgaste físico e emocional, deixando-a mais vulnerável aos acidentes. Em um trabalho realizado num hospital universitário, Balsamo e Felli (2006) encontraram que as mulheres se acidentam mais que os homens, determinando um coeficiente de risco duas vezes maior para as mulheres do que para os homens. Por outro lado, Brandi, Benatti e Alexandre (1998) mostraram uma tendência do profissional do sexo masculino se acidentar mais que as mulheres quando desempenha a mesma função.

A proporção de acidentados é nitidamente menor entre enfermeiros $(21,1 \%)$ quando comparados aos técnicos $(49,8 \%)$ e aos auxiliares $(54,3 \%)$. Isso pode ser explicado pelo fato dos técnicos e dos auxiliares de enfermagem estarem mais expostos a esse tipo de acidente por permanecerem a maior parte do tempo na assistência direta aos pacientes e executarem vários procedimentos invasivos, sendo os materiais perfurocortantes uns dos principais instrumentos de trabalho na prática diária. Além disso, esses profissionais são os que mais manipulam materiais médico-hospitalares e são, em geral, os responsáveis pelo descarte desses materiais. Este argumento é reforçado pelo trabalho realizado por Brandi, Benatti e Alexandre (1998) cujo resultado sugeriu uma relação entre o tipo de atividade executada e a ocorrência do acidente com material biológico, visto que o auxiliar e o técnico de enfermagem são profissionais que têm maior envolvimento no cuidado direto ao paciente.

Neste sentido, entende-se que os enfermeiros, devido ao tipo de atividade, tenham sido menos vítimas de acidentes biológicos, visto que, no contexto do HGP, eles atuam dentro de uma atividade mais administrativa e têm uma atuação direta com o paciente menos intensa que as outras categorias da área. Esse afastamento do enfermeiro como responsável direto da assistência ao paciente também tem sido apontado por outros autores, atitude que implica numa transferência da função assistencial para as outras categorias da enfermagem, sem formação superior, que a exercem com menor eficiência e eficácia. Além disto, é importante ressaltar a questão da formação profissional universitária dos enfermeiros como um fator que, evidentemente contribui para que eles tomem medidas preventivas mais eficazes (RODRIGUES; MAGALHÃES; SALES, 1995; ALMEIDA et al., 2009).

Quanto ao tempo de serviço em relação à ocorrência de acidentes com material biológico, observa-se que a distribuição foi mais ou menos homogênea, exceto em relação ao grupo com menos de 1 ano de trabalho, o menos vitimado por acidentes. Esta menor ocorrência entre os funcionários com menos de 1 ano de atividade pode ser entendida por não fazerem parte da equipe do hospital durante o primeiro ano avaliado e também por ser um número menor de funcionários que aí se enquadram. Canini et al. (2002) encontraram predominância de trabalhadores acidentados com tempo de serviço compreendido entre menos de 1 ano e entre 1 e 5 anos, perfazendo um total de $55,3 \%$. Da mesma forma, Moura, Gir e Canini (2006), em estudo realizado em hospital regional no interior do Estado de Minas Gerais, constataram predomínio de acidentes entre profissionais da enfermagem com menos de 5 anos de serviço. Em estudo realizado por Caixeta e Barbosa-Branco (2005), o tempo de serviço não influenciou na ocorrência de acidentes, mas demonstrou-se um maior coeficiente de acidentes na categoria médica dentre aqueles que possuíam menor tempo de serviço.

Os acidentes com material perfurocortante neste grupo foram mais frequentes do que aqueles por fluidos, respectivamente, $55,6 \%$ e $44,4 \%$. Outros trabalhos também mostram predomínio dos acidentes perfurocortantes, com percentuais que variam de $40 \%$ a 90\% (BREVIDELLI, 1997; SUAZO, 1999; NISHIDI; BENATTI; ALEXANDRE, 2004; CAIXETA; BARBOSA-BRANCO, 2005; ALMEIDA; BENATTI, 2007). Estudo desenvolvido em São Paulo mostrou predomínio dos acidentes perfurocortantes $(87,5 \%)$, sendo o escalpe e a agulha de injeção os objetos responsáveis por 35,0\% dos casos (BALSAMO; FELLI, 2006). Portanto, parte desses acidentes poderia ser evitada pela aplicação das precauções padrão e de outras medidas preventivas, como adequação das caixas de descarte de materiais perfurocortantes e treinamento específico que oriente os trabalhadores da área de saúde sobre os riscos biológicos aos quais estão expostos (WANG et al., 2003). Os acidentes perfurocortantes, pelas suas características intrínsecas, podem traduzir uma situação mais objetiva e, possivelmente, mais assustadora, explicando porque foram mais notificados em relação aos acidentes com fluidos. Por outro lado, alguns estudos demonstram que, dentre os acidentes não notificados, a maioria foi percutânea (NAPOLEÃO, 1999; FACCHIN, 2009).

O levantamento feito no SST da instituição mostrou que foram registrados apenas 64 casos de acidentes com material biológico em todo o período na equipe de enfermagem. Mesmo não se identificando se esses registros eram todos referentes aos participantes da atual pesquisa, fica clara a existência de 
importante subnotificação. Possivelmente, esta subnotificação encontra explicação no desconhecimento dos funcionários em como proceder corretamente no momento de notificar. Alguns podem considerar que uma queixa ou um relato verbal tenha o valor de uma notificação. Outra possibilidade é de que notificações verbais não tenham sido adequadamente consideradas e formalizadas no SST. Sendo assim, é possível entender que o processo informação-decisão-ação, que caracteriza a vigilância epidemiológica, tenha falhado já no primeiro item, que é a informação.

Marziale, Nishimura e Ferreira (2004), em estudo que avaliou trabalhadores de enfermagem de quatro hospitais da região de Ribeirão Preto (SP), identificaram disparidade no número de encaminhamentos de trabalhadores ao serviço de especialidades e o número de trabalhadores realmente atendidos, concluindo que existem fatores que impedem a execução de uma avaliação especializada após a ocorrência de acidentes. As autoras propõem que seja feita uma reavaliação na forma de encaminhamento para a unidade notificadora. A burocracia também foi um dos achados impeditivos na eficácia da notificação no trabalho de Nishide et al. (2004).

O descuido em notificar não é um problema local. Embora o acidente por material perfurocortante seja, ao longo do tempo, causador de doença ou até de morte do trabalhador, tanto o empregador, como o empregado acidentado o menosprezam. A subnotificação de acidentes tem sido determinada em vários estudos, com prevalências bastante variadas, chegando a valores tão altos quanto 91\%. Em geral, os principais motivos apontados para não se notificar são a falta de conhecimento sobre como fazê-lo, o excesso de burocracia, medo de punição pela chefia imediata e atribuição de baixo risco ao acidente (BRANDI; BENATTI; ALEXANDRE, 1998; NAPOLEÃO, 1999; CANINI, 2000; FACCHIN, 2009; CANALLI ; MORIYA; HAYASHIDA, 2010).

Muitos trabalhadores julgam estar isentos de riscos quando se acidentam com material estéril. Entretanto, mesmo sem risco de contaminação, o acidente preocupa, pois evidencia um erro técnico no trabalho, podendo ocorrer em outra atividade na qual a agulha esteja contaminada.
Esses resultados implicam em reflexões e questionamentos acerca da falta de conhecimento ou de conscientização dos trabalhadores quanto aos riscos de exposição ocupacional aos agentes do sangue e da importância de receber o esquema completo da vacina contra a hepatite B como medida de proteção. A AIDS foi a doença declarada como a que mais temor desperta quando de uma exposição acidental, sendo a primeira citada por $57,8 \%$ dos entrevistados. Por ser uma doença incurável, de grande impacto para a população mundial, é preciso que os trabalhadores de saúde estejam cada vez mais habilitados para atuarem preventivamente.

Apesar do risco de adquirir infecções ao cuidar de pacientes contaminados por algum agente microbiológico patogênico ser bem conhecido, somente após a descoberta do vírus da imunodeficiência humana (HIV), como agente da Síndrome da Imunodeficiência Adquirida (AIDS) e a elucidação da sua forma de transmissão é que as organizações estatais desencadearam esforços para diminuir as consequências dos acidentes com exposição ocupacional aos fluidos orgânicos potencialmente contaminados (ALMEIDA; BENATTI, 2007).

Os resultados desse trabalho deixam evidente a necessidade de se fazer um treinamento com a equipe de enfermagem no sentido de diminuir os riscos de acidentes com material biológico relacionados ao trabalho, bem como difundir a cultura da notificação. Neste sentido, o profissional da saúde deve ser treinado para identificar situações de risco de acidentes e propor alternativas de proteção à sua própria saúde e à dos demais profissionais, e a instituição deve tomar todas as medidas que facilitem a execução desses objetivos. Estudos posteriores devem ser realizados no sentido de verificar se os treinamentos propostos modificam a incidência dos acidentes com material biológico nas diferentes categorias da equipe de enfermagem e avaliar a ocorrência dos acidentes em outros profissionais da área de saúde, tanto no HGP, como em outros hospitais regionais. Igualmente, interessa saber a influência de cursos de treinamento na diminuição das subnotificações.

\section{Contribuições de autoria}

Ambos os autores contribuíram de maneira substancial tanto no projeto, como em sua execução e participaram igualmente da redação deste trabalho. 


\section{Referências}

ALMEIDA, A. N. G. et al. Risco Biológico entre os trabalhadores de enfermagem. Revista Enfermagem UERJ, Rio de Janeiro, v. 17, n. 4, p. 595-600, 2009.

ALMEIDA, C. A. F; BENATTI, M. C. C. Exposição ocupacional por fluidos corpóreos entre trabalhadores da saúde e sua adesão à quimioprofilaxia. Revista $d a$ Escola de Enfermagem da USP, São Paulo, v. 41, n. 1, p. 120-126, 2007.

BALSAMO, A. C.; BARRIENTO, D. S.; ROSSI, J. C. B. Estudo retrospectivo dos acidentes de trabalho com exposição a líquidos corporais humanos ocorridos nos funcionários de um hospital universitário. Revista de Medicina do Hospital Universitário da Universidade de São Paulo, São Paulo, v. 10, n. 1, p. 39-45, 2000.

BALSAMO, A. C.; FELLI, V. E. A. Estudo sobre os acidentes de trabalho com exposição aos líquidos corporais humanos em trabalhadores da saúde de um hospital universitário. Revista Latino-Americana de Enfermagem, Ribeirão Preto, v. 14, n. 3, p. 346-353, 2006.

BRAGA, A. S. P. Prevalência de marcadores sorológicos do vírus da Hepatite $B$ em cirurgiões-dentistas após vacinação. 2005. 99 f. Dissertação (Mestrado)Universidade Federal da Bahia, Salvador, 2005.

BRANDI, S.; BENATTI, M. C. C.; ALEXANDRE, N. M. C. Ocorrência de acidente de trabalho por material perfuro-cortante entre trabalhadores de enfermagem de um Hospital Universitário da cidade de Campinas. Revista da Escola de Enfermagem da USP, v. 32, n. 2, p. 124-133, 1998.

BRASIL. Ministério da Saúde. Secretaria de Políticas de Saúde. Coordenação Nacional de DST/AIDS. Controle de Infecção e a prática odontológica em tempos da AIDS: manual e condutas. Brasília, DF, 2000 .

- Ministério da Saúde. Doenças relacionadas ao trabalho: manual de procedimentos para serviços de saúde. Brasília, DF, 2001. (Série A. Normas e Manuais Técnicos, n. 114)

. Ministério da Saúde. Recomendações para atendimento e acompanhamento de exposição ocupacional a material biológico: HIV e Hepatites B e C. Brasília, DF, 2004.

BREVIDELLI, M. M. Exposição ocupacional ao vírus da AIDS e da hepatite B: análise da influencia das crenças em saúde sobre a prática de reencapar agulhas. 1997. 157 f. Dissertação (Mestrado em Enfermagem)-Escola de Enfermagem da USP, São Paulo, 1997.

CAIXETA, R. B.; BARBOSA-BRANCO, A. Acidente de trabalho com material biológico, em profissionais de saúde de hospitais públicos do Distrito Federal, Brasil. 2002/2003. Cadernos de Saúde Pública, São Paulo, v. 21, n. 3, p. 737-746, 2005.
CANALLI, R. T. C. Acidentes com material biológico entre estudantes de enfermagem de um município do interior paulista. 2008. 124 f. Dissertação (Mestrado em Enfermagem)-Escola de Enfermagem de Ribeirão Preto, Universidade de São Paulo, Ribeirão Preto, 2008.

CANALLI, R. T. C.; MORIYA, T. M.; HAYASHIDA, M. Acidentes com material biológico entre estudantes de enfermagem. Revista Enfermagem UERJ, Rio de Janeiro, v. 18, n. 2, p. 259-264, 2010.

CANINI, S. R. M. S. Situações de risco para transmissão de patógenos veiculados pelo sangue entre a equipe de enfermagem de um Hospital Universitário do interior paulista. 2000. 122 f. Dissertação (Mestrado em Enfermagem)-Escola de Enfermagem de Ribeirão Preto, Universidade de São Paulo, Ribeirão Preto, 2000.

CANINI, S. R. M. S. et al. Acidentes perfurocortantes entre trabalhadores de enfermagem de um hospital universitário do interior paulista. Revista LatinoAmericana de Enfermagem, Ribeirão Preto, v. 10, n. 2, p. 172-178, 2002.

CENTERS FOR DISEASE CONTROL AND

PREVENTION. Update U.S. Health Service Guidelines for the Management of Occupational Exposures to HIV and Recommendatios for Postexposure Prophylaxis. Atlanta: CDC, 2005. (Recommendations and Reports: Mordibity and Mortality Weekly Report, v. 54, n. RR- 9)

CIORLIA, L. A. S.; ZANETTA, D. M. T. Significado epidemiológico dos acidentes de trabalho com material biológico: Hepatites B e C em profissionais da área de saúde. Revista Brasileira de Medicina do Trabalho, Belo Horizonte, v. 2, n. 3, p. 191-199, 2004.

FACCHIN, L. T. Prevalência de subnotificação de acidentes com material biológico pela equipe de enfermagem de um hospital de urgência. 2009. 98 f. Dissertação (Mestrado em Enfermagem)-Escola de Enfermagem de Ribeirão Preto, Universidade de São Paulo, Ribeirão Preto, 2009.

GALON, T.; ROBAZZI, M. L. C. C.; MARZIALE, M. H. P. Acidentes de trabalho com material biológico em hospital universitário de São Paulo. Revista Eletrônica de Enfermagem, Goiânia, v. 10, n. 3, p. 673-85, 2008. Disponível em: < http://www.fen.ufg.br/revista/v10/n3/ v10n3a13.htm>. Acesso em: 05 maio 2009.

GARNER, J. S. CDC Guideline for prevention of surgical wound infections. American Journal Infection Control, New York, v. 14, n. 2, p. 71-82, 1996.

GUILARDE, A. O et al. Acidentes com material biológico entre profissionais de hospital universitário em Goiânia. Revista de Patologia Tropical, Goiânia, v. 39, n. 2, p. 131-136, 2010.

MARZIALE, M. H. P.; NISHIMURA, K. Y. N.; FERREIRA, M. M. Riscos de contaminação ocasionados por acidentes de trabalho com material 
perfuro-cortante entre trabalhadores de enfermagem. Revista Latino-Americana de Enfermagem, Ribeirão Preto, v. 12, n. 1, p. 36-42, 2004.

MOURA, J. P.; GIR, E.; CANINI, S. R. M. S. Acidentes ocupacionais com material perfurocortante em um Hospital Regional de Minas Gerais, Brasil. Revista Ciência y Enfermeria, Concepcion, v. 12, n. 1, p. 29-37, 2006.

NAPOLEÃO, A. M. Causas de subnotificação de acidentes de trabalho: visão dos trabalhadores de um hospital do interior paulista. 1999. 115 f. Dissertação (Mestrado em Enfermagem)-Escola de Enfermagem de Ribeirão Preto, Universidade de São Paulo, Ribeirão Preto, 1999.

NISHIDE, V. M.; BENATTI, M. C. C.; ALEXANDRE, N. M. C. Ocorrência de acidente do trabalho em uma unidade de terapia intensiva. Revista Latino-Americana de Enfermagem, Ribeirão Preto, v. 12, n. 2, p. 204-211, 2004.

RAPPARINI, C. Occupational HIV infection among health care workers exposed to blood and body fluids in Brazil. American Journal Infection Control, New York, v. 34, n. 4, p. 237-240, 2002.

RAPPARINI, C. et al. Occupational exposures to bloodborne pathogens among healthcare workers in Rio de Janeiro, Brazil. Journal Hospital Infection, London, v. 65, n. 2, p. 131-137, 2007.

RESENDE, M. R.; FORTALEZA, C. M. C. B. Risco ocupacional entre profissionais da área de saúde e medidas de proteção. In: COLIBRINI, M. R. C.; FIGUEIREDO, R. M.; PAIVA, M. C. (Org.). Leito-dia em AIDS: uma experiência multiprofissional. São Paulo: Atheneu, 2001. p. 139-157.
RODRIGUES, A. B.; MAGALHÃES, M. B. B.; SALES, S. M. M. A questão do vírus da imunodeficiência humana e a autoproteção no trabalho. Revista Brasileira de Enfermagem, Brasília, DF, v. 48, n. 3, p. 272-285, 1995.

SHIMUZU, H. E.; RIBEIRO, E. J. G. Ocorrência de acidente de trabalho por materiais perfurocortantes e fluídos biológicos em estudantes e trabalhadores da saúde de um hospital escola de Brasília. Revista Escola Enfermagem, São Paulo, v. 36, n. 4, p. 367-375, 2002.

SPAGNUOLO, R. S.; BALDO, R. C. S.; GUERRINI, I. A. Epidemiological analysis of accidents with biological material reported to the worker's health reference center in Londrina-PR. Revista Brasileira de Epidemiologia, São Paulo, v. 11, n. 2, p. 315-323, 2008.

SOERENSEN, A. A. et al. Acidentes com material biológico em profissionais do atendimento préhospitalar móvel. Revista de Enfermagem UERJ, Rio de Janeiro, v. 17, n. 2, p. 234-239, 2009.

SUAZO, S. U. V. Contribuição ao estudo sobre acidentes de trabalho que acometem as trabalhadoras de enfermagem em hospitais chilenos. 1999. $184 \mathrm{f}$. Dissertação (Mestrado em Enfermagem)-Escola de Enfermagem de Ribeirão Preto, Ribeirão Preto, 1999.

TEIXEIRA, M.; SANTOS, M. V. Responsabilidade no controle de infecção. Revista da APCD, São Paulo, v. 53, n. 3, p. 177-189, 1999.

WANG, H. et al. A training programme for prevention of occupational exposure to bloodborne pathogens: impact on knowledge, behaviour and incidence of needle stick injuries among student nurses in Changsha, People's Republic of China. Journal of Advance Nursing, Malden, v. 41, n. 2, p. 187-194, 2003. 